Introduction: The treatment outcomes of patients with advanced/metastatic melanoma were poor before the use of new therapeutic options.

Material and methods: A retrospective analysis was conducted among 287 patients with unresectable stage III and stage IV melanoma treated at the Maria Sklodowska-Curie National Research Institute of Oncology Cracow Branch, from 2013 to 2019. All enrolled patients were treated with immunotherapy (IT; consisting of pembrolizumab/nivolumab, or ipilimumab) or target therapy (TT; consisting of vemurafenib \pm cobimetinib or dabrafenib \pm trametinib) in at least one treatment line.

Results: mutation was detected in 152 (55\%) patients. In general, the majority of patients (92\%) were in very good or good condition (Eastern Cooperative Oncology Group [ECOG] 0 or 1$)$. Brain metastasis was detected in $64(22 \%)$ patients. Median OS and PFS in the experimental group from the beginning of the first-line treatment were 14.9 and 6.7 months, respectively. Across the study population, as a first-line treatment patients received IT, TT as well as $\mathrm{CHT}$, and the median OS was 19.2, 12.6 and 15.9 months, respectively. Multivariate analysis confirmed that normal LDH levels, no brain metastases, ECOG 0, and objective response to the treatment were strong predictors of longer OS. For PFS, absence of brain metastases, ECOG 0 , and treatment response were found to be predictive factors on multivariate analysis.

Conclusions: The administration of new therapies for the treatment of patients with advanced/disseminated melanoma significantly prolonged survival in this group of patients. Nevertheless, further studies should be conducted to assess the effectiveness of various sequences of treatment.

Key words: melanoma, metastases, immunotherapy, targeted therapy, prognostic factors, real-world data.

Contemp Oncol (Pozn) 2020; 24 (2): 118-124 DOI: https://doi.org/10.5114/wo.2020.97607

\section{Real-world treatment practice in patients with advanced melanoma}

\author{
Bożena Cybulska-Stopa, Karolina Piejko, Renata Pacholczak, \\ Małgorzata Domagała-Haduch, Anna Drosik-Kwaśniewska, Janusz Rolski, \\ Patrycja Wiktor-Mucha, Tomasz Zemełka
}

Clinical Oncology Department, Maria Sklodowska-Curie National Research Institute of Oncology Krakow Branch, Krakow, Poland

\section{Introduction}

The treatment outcomes of patients with advanced/metastatic melanoma were poor before the use of new therapeutic options. The median overall survival period via a standard single agent or multidrug chemotherapy (CTH) was 6-11 months [1-5]. The development of new types of therapies such as immunotherapy (IT) and targeted therapy (TT) has significantly improved the outcomes of patients with advanced/disseminated melanoma. At present, the median aggregate survival period observed in clinical trials using IT or TT is over three years [6-12].

Immunotherapy uses immune checkpoint inhibitors (ICls), which include anticytotoxic T-lymphocyte antigen 4 (anti-CTLA-4) antibodies - ipilimumab and antibodies against the programmed cell death-1 (anti-PD-1) inhibitors - nivolumab and pembrolizumab [13]. Targeted therapy includes BRAF proto-oncogene serine/threonine kinase inhibitors (BRAFi; vemurafenib, dabrafenib, and encorafenib) and MEK inhibitors (MEKi; cobimetinib, trametinib, and binimetinib) [14].

In Poland, IT or TT treatment of patients with melanoma was initiated and refunded by the National Health Fund (NFZ) in 2013 as part of the national drug program. Vemurafenib was the first of the new class of drugs to be refunded under the national drug program (from March 1, 2013). The next few drugs to be refunded were: ipilimumab, which was used as a second-line treatment in patients with advanced/metastatic melanoma (from March 1, 2014), dabrafenib (from July 1, 2015), and cobimetinib and trametinib (from 2017) as a supplement to vemurafenib and dabrafenib therapy, respectively. Nivolumab and pembrolizumab have been used and refunded from June 1, 2016. Initially, BRAFi/MEKi were only refunded prior to anti-PD-1 therapy. Since 2017, BRAFi/MEKi have been reimbursed in the first, second, and subsequent treatment lines and also after anti-PD-1 therapy. Therefore, from 2017, anti-PD-1 was used as first-line treatment for BRAF-mutated patients.

This study aims at analysing the treatment outcomes of patients with advanced/disseminated melanoma through new therapies under the national drug program.

\section{Material and methods}

A retrospective analysis was conducted for 287 patients with unresectable stage III and stage IV melanoma treated at the Maria Sklodowska-Curie National Research Institute of Oncology Krakow Branch, from 2013 to 2019. Moreover, this retrospective non-interventional study did not require any additional approval from an ethical authority. All the analysed data were gathered as part of routine diagnosis and treatment. Patients were diagnosed and treated in line with national guidelines and agreements. Treatments were covered according to the NFZ reimbursement regulations (Poland) outlined 
by the Agency for Health Technology Assessment and Tariff System (AOTMiT). All the patients signed an informed consent form for the treatment as a standard operating procedure in our hospital. The patients who participated in the clinical trials were excluded from the analysis.

All the enrolled patients were treated with IT (comprising pembrolizumab/nivolumab/ipilimumab) or TT (comprising vemurafenib \pm cobimetinib or dabrafenib \pm trametinib) in at least one treatment line. All the patients were included in the treatment according to the inclusion criteria of the drug programs. Data on age, sex, location of the primary lesion, presence or absence of BRAF mutation, stage of the disease, and type of therapy used in the first, second, third, and subsequent lines were recorded. Information on the stage of the disease, location of metastatic lesions, LDH level, and Eastern Cooperative Oncology Group (ECOG) performance status were collected at the beginning of first-line systemic therapy.

\section{Statistical analysis}

The primary aims of the study were progression-free survival (PFS), overall survival (OS), overall response rate $(\mathrm{ORR})$, and disease control rate (DCR) defined by the re- sponse evaluation criteria in solid tumours (RECIST) 1.1. PFS and OS were measured from the first administration of medication until disease progression according to RECIST, death, or last documented/reported visit. Patients who were alive at the end of the study period were examined on the date of the last follow-up. The Kaplan-Meier method was used to calculate PFS and OS survival curves, and the log-rank test was used to compare these measures. The multivariate analyses, performed using Cox's proportional hazards model, were used to evaluate connections between predictive factors (patients and treatment characteristics) as well as PFS and OS. The differences were considered statistically significant if the $p$-values were $<0.05$. All statistical analyses were performed using Statistica 12 software.

\section{Baseline characteristics}

The median age in the study group was 63 years (range: 18-94 years), among whom 119 (42\%) patients were above 65 years of age. Men represented $60 \%$ of the total population. The main location of the primary lesion was in the skin (85\%), and less often in a mucous membrane or an unknown primary site ( $4 \%$ and $11 \%$, respectively). BRAF mutation was

Table 1. Patient characteristics according to therapy regimen.

\begin{tabular}{|c|c|c|c|c|c|c|c|}
\hline \multirow[t]{3}{*}{ Characteristics } & & \multicolumn{5}{|c|}{ Therapy regimen } & \multirow{3}{*}{$\begin{array}{c}\text { Total } \\
n=287(\%)\end{array}$} \\
\hline & & \multicolumn{2}{|c|}{ Immunotherapy } & \multirow{2}{*}{$\begin{array}{c}\text { Targeted therapy } \\
n=111(\%)\end{array}$} & \multicolumn{2}{|c|}{ Chemotherapy } & \\
\hline & & $\begin{array}{c}\text { Total } \\
n=133(\%)\end{array}$ & $\begin{array}{c}B R A F \text {-mutated } \\
n=28(\%)\end{array}$ & & $\begin{array}{c}\text { Total } \\
n=43(\%)\end{array}$ & $\begin{array}{c}B R A F \text {-mutated } \\
n=13(\%)\end{array}$ & \\
\hline \multirow[t]{3}{*}{ Age (years) } & Median (range) & 66 (27-94) & $60(27-86)$ & $60(25-82)$ & $65(18-85)$ & $57(40-77)$ & 63 (18-94) \\
\hline & $\leq 65$ & $64(48)$ & $20(71)$ & $78(70)$ & $26(60)$ & $9(69)$ & $168(58)$ \\
\hline & $>65$ & $69(52)$ & $8(29)$ & $33(30)$ & $17(40)$ & $4(31)$ & $119(42)$ \\
\hline \multirow[t]{2}{*}{ Gender } & Male & $85(64)$ & $19(68)$ & $60(54)$ & $28(65)$ & $8(62)$ & $173(60)$ \\
\hline & Female & $48(36)$ & $9(32)$ & $51(46)$ & $15(35)$ & $5(38)$ & $114(40)$ \\
\hline \multirow[t]{2}{*}{$B R A F$ mutation ${ }^{*}$} & No & $102(77)$ & - & - & $24(65)$ & - & $126(45)$ \\
\hline & Yes & $28(23)$ & $28(100)$ & $111(100)$ & $13(35)$ & $13(100)$ & $152(55)$ \\
\hline \multirow[t]{2}{*}{ Brain metastasis } & No & $113(85)$ & $25(89)$ & $71(64)$ & $39(91)$ & $10(77)$ & $223(78)$ \\
\hline & Yes & $20(15)$ & $3(11)$ & $40(36)$ & $4(9)$ & $3(23)$ & $64(22)$ \\
\hline \multirow{4}{*}{$\begin{array}{l}\text { Stage according } \\
\text { to AJCC } 8^{\text {th }} \\
\text { edition }\end{array}$} & M1a & $37(28)$ & $7(25)$ & $14(13)$ & $12(28)$ & $4(31)$ & $63(22)$ \\
\hline & M1b & $26(20)$ & $6(21)$ & $17(15)$ & $13(30)$ & $1(8)$ & $56(20)$ \\
\hline & M1c & $50(37)$ & $12(43)$ & $40(36)$ & $14(33)$ & $5(38)$ & $104(36)$ \\
\hline & M1d & $20(15)$ & $3(11)$ & $40(36)$ & $4(9)$ & $3(23)$ & $64(22)$ \\
\hline \multirow{2}{*}{$\begin{array}{l}\text { Count of location } \\
\text { of metastasis }\end{array}$} & $\leq 2$ & $83(62)$ & $5(18)$ & $51(46)$ & $25(58)$ & $3(23)$ & $159(55)$ \\
\hline & $>2$ & $50(38)$ & $23(82)$ & $60(54)$ & $18(42)$ & $10(77)$ & $128(45)$ \\
\hline \multirow[t]{2}{*}{$\mathrm{LDH}$} & Normal & $79(61)$ & $21(75)$ & $41(38)$ & $31(74)$ & $8(62)$ & $151(54)$ \\
\hline & Increased & $50(39)$ & $7(25)$ & $67(62)$ & $11(26)$ & $5(38)$ & $129(46)$ \\
\hline \multirow[t]{3}{*}{ ECOG } & 0 & $14(11)$ & $3(11)$ & $12(11)$ & $2(5)$ & $2(15)$ & $28(10)$ \\
\hline & 1 & $110(85)$ & $24(86)$ & $84(76)$ & 38 (90) & $10(77)$ & $232(82)$ \\
\hline & $>1$ & $6(4)$ & $1(3)$ & 15 (13) & $2(5)$ & $1(8)$ & $23(8)$ \\
\hline \multirow[t]{3}{*}{ Location } & Skin & 107 (84) & $24(89)$ & $91(88)$ & $36(84)$ & $11(85)$ & $234(85)$ \\
\hline & Mucous & $11(8)$ & $1(3)$ & 0 & $1(2)$ & 0 & $12(4)$ \\
\hline & Unknown & $10(8)$ & $2(8)$ & $12(12)$ & $6(14)$ & $2(15)$ & $28(11)$ \\
\hline
\end{tabular}

LDH - lactate dehydrogenase, ECOG/PS - Eastern Cooperative Oncology Group/Performance status * BRAF mutation unknown for 9 patients 
detected in 152 (55\%) patients. In general, the majority of patients (92\%) were in very good or good condition (ECOG 0 or 1). Brain metastasis was detected in 64 (22\%) patients. Table 1 summarises the baseline characteristics of patients.

\section{Types and sequences of the treatments}

In the first line, IT was administered to 133 (46\%) patients, TT to 111 (39\%) patients, and CTH to 43 (15\%) patients. In the entire group, 138 (48\%) patients qualified for the second line of therapy: 114 (82\%) patients received IT, 13 (10\%) received TT, while the remaining 11 (8\%) received CTH. Only 34 (13\%) of the enrolled patients received the third line of therapy: IT was prescribed to two (6\%),
TT to 10 (30\%), and CTH to 22 (64\%) of the patients. The fourth-line treatment was limited to seven (2\%) of the patients who received CTH $(n=5,72 \%)$, IT $(n=1,14 \%)$, and hormone therapy (HT; $n=1,14 \%)$. The fifth-line treatment (dacarbazine chemotherapy) was provided to only one patient. Figure 1 displays the treatment sequences for the first, second, third, and fourth lines for BRAF-mutated (Fig. 1A) and BRAF-wild type (Fig. 1B) patients.

\section{Treatment outcomes corresponding to the line of} treatment

Median OS and PFS in the study group from the beginning of the first-line treatment were 14.9 and 6.7 months,

A

BRAF+

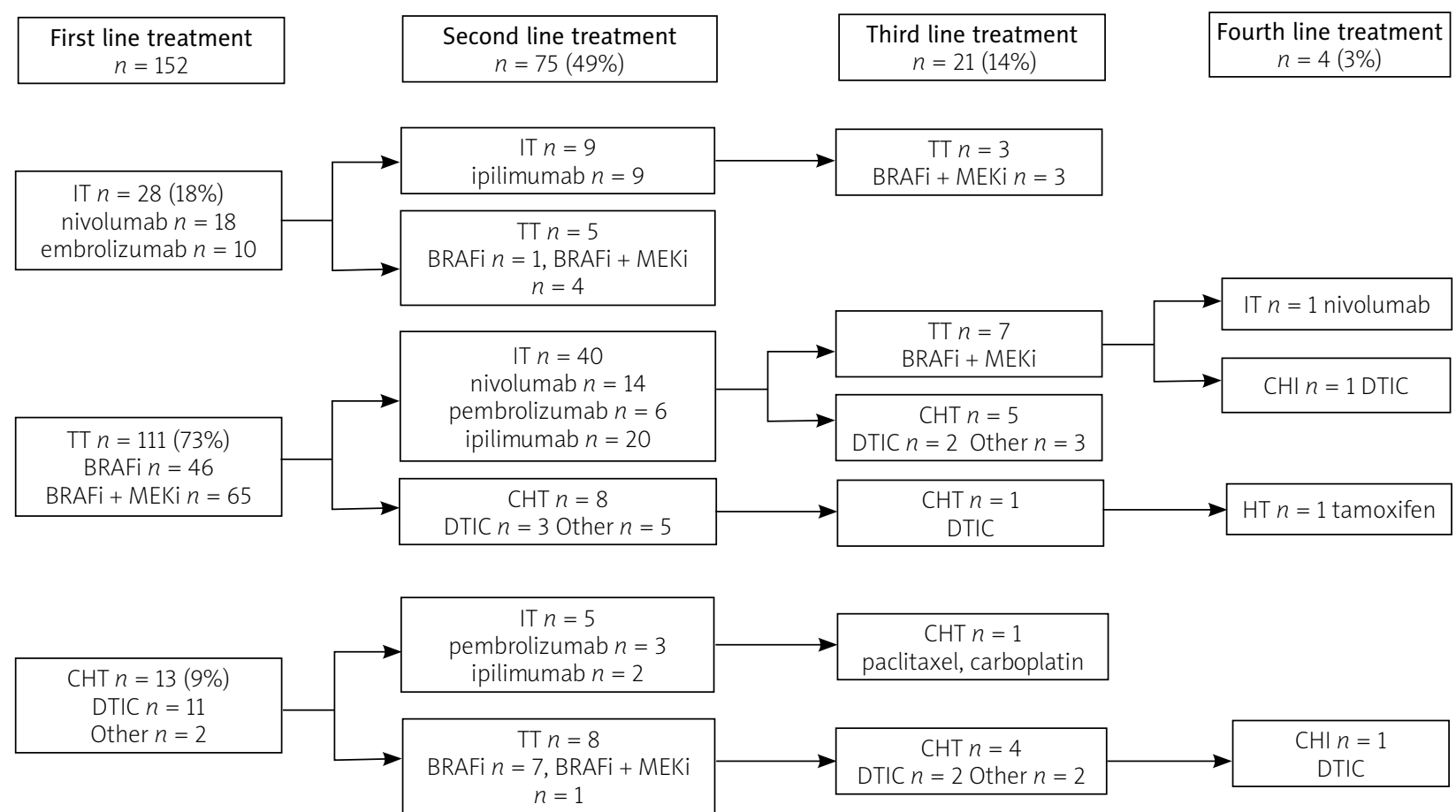

B

$B R A F-*$

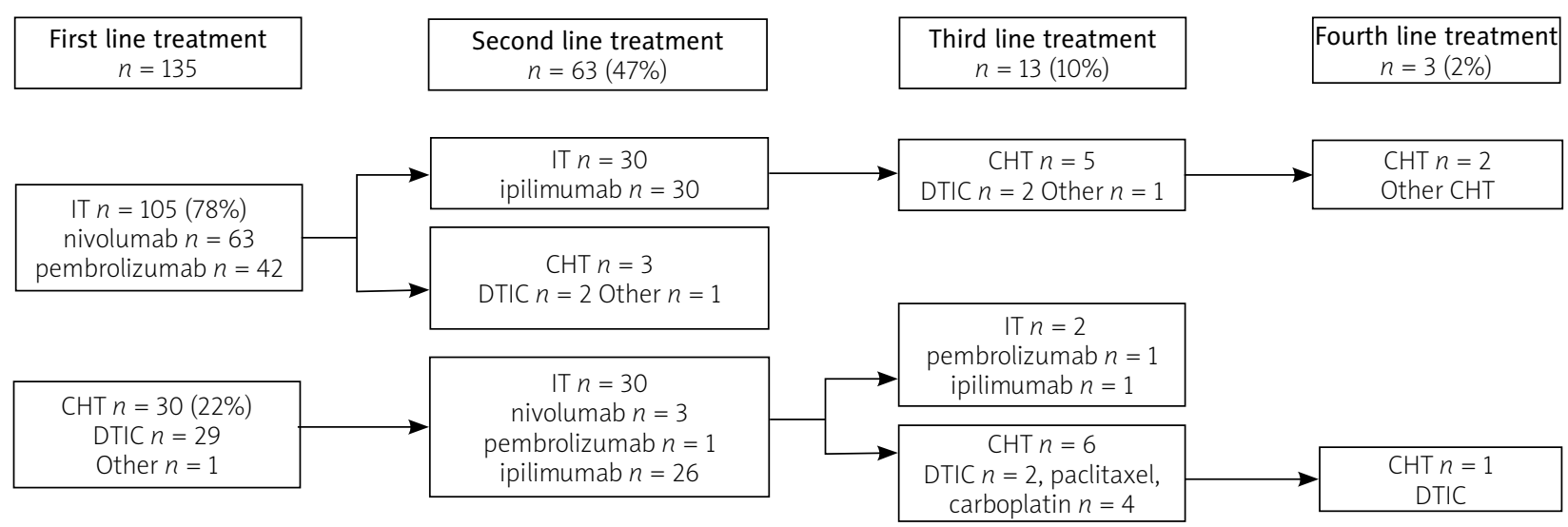

* BRAF mutation unknown for 9 (7\%) patients

Fig. 1. Types of treatment in subsequent lines of therapy for BRAF-mutated (A) and BRAF-wild type (B) patients (IT - immunotherapy, TT - targeted therapy, CHT - chemotherapy, DTIC - dacarbazine) 
respectively (Fig. 2A and 2B). In the second line, OS and PFS were 7.2 and 3.1 months, respectively, and in the third line they were 5.5 and 3.9 months, respectively (Fig. 2C). Based on the first-line treatment, the median OS was 19.2, 12.6, and 15.9 months for IT, TT, and CTH, respectively. The median follow-up was 12.6 months (range 0.2-85.9). However, it was longer for the CTH subgroup at 15.0 (range 1.2-85.9) months compared to IT and 11.7 (range 0.2-36.5) months and TT at 11.9 (range 0.2-62.5) months. Table 2 represents the survival outcomes and response rates based on the types and sequences of the therapy.
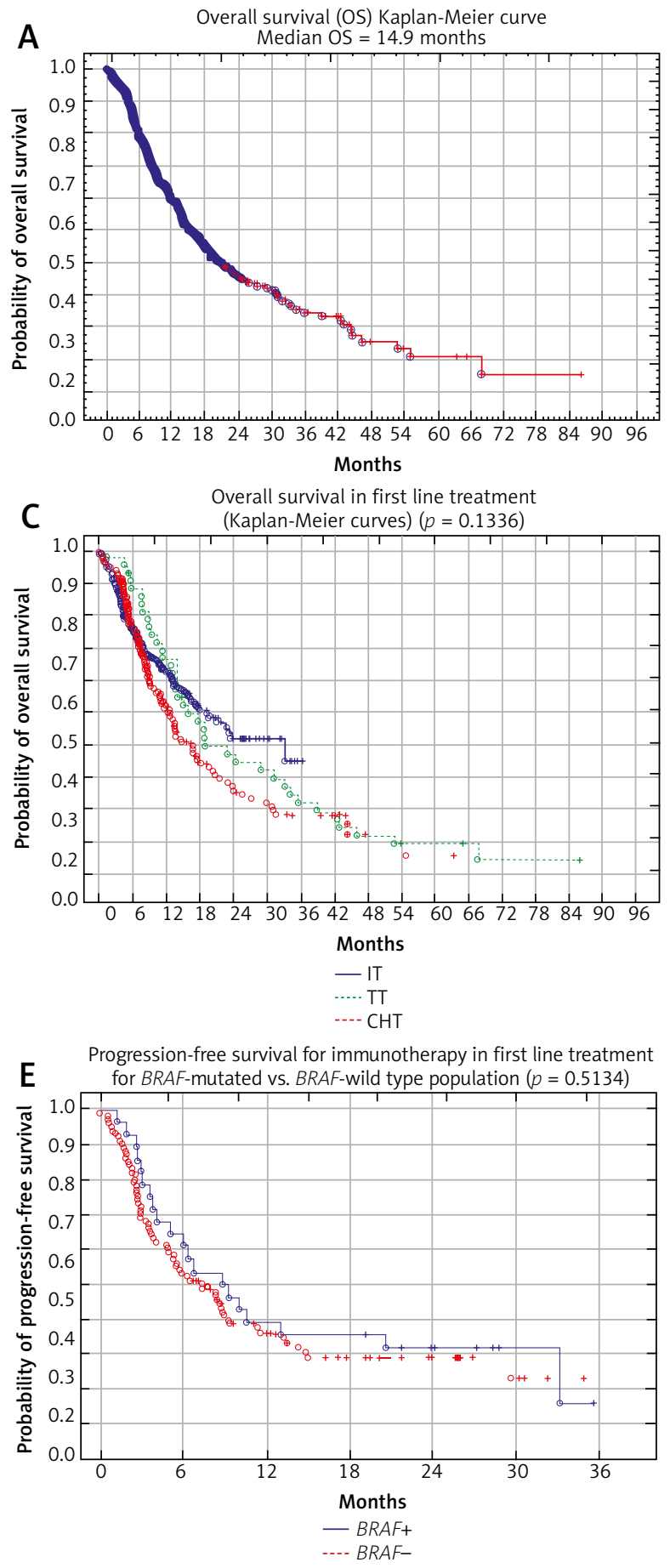

Treatment results in the BRAF-mutated and BRAFwild type population

The median OS in the BRAF-mutated and BRAF-wild type populations were 14.2 and 14.9 months, respectively, and there was no statistically significant difference ( $p=$ 0.7972). There was also no statistically significant difference in PFS in these groups ( $p=0.3663)$.

The median OS for IT in first-line treatment in the BRAF-mutated population was not reached, and in the $B R A F$-wild type population it was 14.8 months. There was a statistically significant difference between the groups

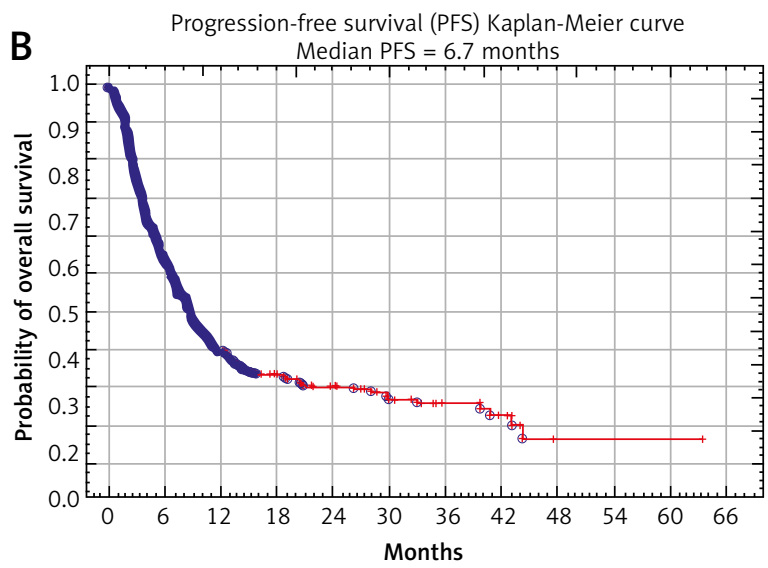

Overall survival for immunotherapy in first line treatment for

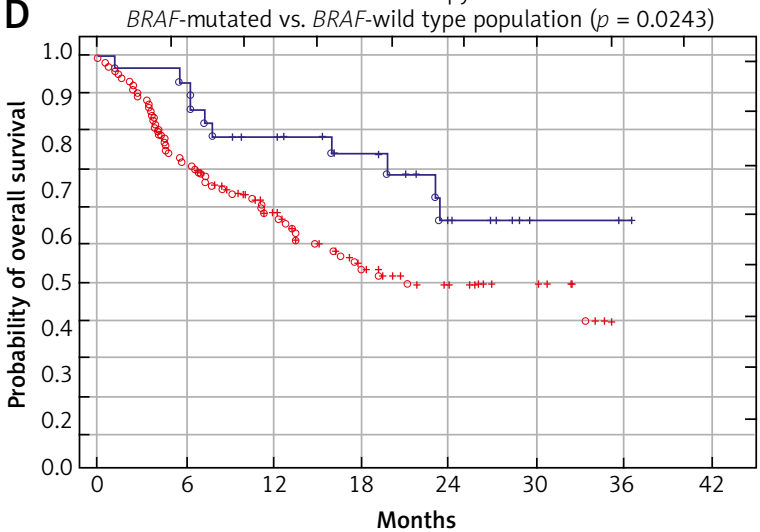

- BRAF+

Fig. 2. Kaplan-Meier curves for overall survival in first-line treatment (A), progression-free survival in first-line treatment (B), overall survival stratified by type of first-line treatment (C), overall survival (D), and progression-free survival (E) for immunotherapy in first-line treatment for BRAF-mutated vs. BRAF-wild type population (IT - immunotherapy, TT - targeted therapy, CHT - chemotherapy) 
Table 2. Results of treatment depending on the type of therapy and treatment line

\begin{tabular}{|c|c|c|c|c|}
\hline Type of therapy & IT & TT & CHT & Total \\
\hline First-line therapy & $n=133$ & $n=111$ & $n=43$ & $n=287$ \\
\hline $\begin{array}{l}\text { Median OS (months) } \\
p=0.1336\end{array}$ & 19.2 & 12.6 & 15.9 & 14.9 \\
\hline 6-month OS (\%) & 75 & 77 & 86 & 77 \\
\hline 1-year OS (\%) & 50 & 50 & 65 & 52 \\
\hline 2-year OS (\%) & 18 & 22 & 35 & 22 \\
\hline $\begin{array}{l}\text { Median PFS (months) } \\
p=0.208\end{array}$ & 7.5 & 7.3 & 3.8 & 6.7 \\
\hline CR (\%) & 3 & 5 & 0 & 4 \\
\hline PR (\%) & 33 & 59 & 9 & 39 \\
\hline $\mathrm{SD}(\%)$ & 35 & 24 & 61 & 34 \\
\hline $\mathrm{ORR}=\mathrm{CR}+\mathrm{PR}(\%)$ & 36 & 64 & 9 & 43 \\
\hline$D C R=C R+P R+S D(\%)$ & 71 & 88 & 70 & 77 \\
\hline PD (\%) & 29 & 12 & 30 & 23 \\
\hline Second-line therapy & $n=114$ & $n=13$ & $n=11$ & $n=138$ \\
\hline $\begin{array}{l}\text { Median OS (months) } \\
p=0.1585\end{array}$ & 7.2 & 9.9 & 3.7 & 7.2 \\
\hline 6-month OS (\%) & 51 & 77 & 30 & 52 \\
\hline 1-year OS (\%) & 29 & 38 & 0 & 27 \\
\hline 2-year OS (\%) & 9 & 0 & 0 & 7 \\
\hline $\begin{array}{l}\text { Median PFS (months) } \\
p=0.0422\end{array}$ & 3.0 & 4.5 & 2.4 & 3.1 \\
\hline CR $(\%)$ & 2 & 0 & 0 & 1 \\
\hline PR (\%) & 12 & 69 & 0 & 17 \\
\hline SD (\%) & 60 & 23 & 45 & 55 \\
\hline $\mathrm{ORR}=\mathrm{CR}+\mathrm{PR}(\%)$ & 14 & 69 & 0 & 18 \\
\hline$D C R=C R+P R+S D(\%)$ & 74 & 92 & 45 & 73 \\
\hline PD (\%) & 26 & 8 & 55 & 27 \\
\hline Third-line therapy & $n=2$ & $n=10$ & $n=22$ & $n=34$ \\
\hline Median OS (months) & 4.6 & 6.5 & 4.8 & 5.5 \\
\hline 6-month OS (\%) & 0 & 60 & 41 & 44 \\
\hline 1-year OS (\%) & 0 & 20 & 4 & 9 \\
\hline 2-year OS (\%) & 0 & 0 & 0 & 0 \\
\hline Median PFS (months) & 4.3 & 4.4 & 2.6 & 3.9 \\
\hline CR (\%) & 0 & 0 & 0 & 0 \\
\hline PR (\%) & 0 & 80 & 5 & 27 \\
\hline $\mathrm{SD}(\%)$ & 50 & 20 & 27 & 27 \\
\hline $\mathrm{ORR}=\mathrm{CR}+\mathrm{PR}(\%)$ & 50 & 100 & 32 & 27 \\
\hline$D C R=C R+P R+S D(\%)$ & 50 & 100 & 32 & 54 \\
\hline PD (\%) & 50 & 0 & 68 & 46 \\
\hline
\end{tabular}

OS - overall survival, PFS - progression-free survival, $C R$ - complete response, $P R$ - partial response, $S D$ - stable disease, $P D$ - progression of disease, $O R R$ overall response rate, $D C R$ - disease control rate

$(p=0.0243)$ (Fig. 2D). However, no significance was demonstrated for PFS ( $p=0.5134)$ (Fig. 2E). The median OS for CHT in first-line treatment in the BRAF-mutated and in the BRAF-wild type population was 15.1 and 14.3 months, respectively, and there was no statistically significant difference ( $p=0.9167)$. However, PFS was longer in the BRAF-mutated population, and there was statistically significant difference ( $p=0.0237)$.

\section{Univariate and multivariate analyses of the experimental group}

A statistically significant positive effect on OS and PFS at the start of the first line of treatment were as follows: the absence of metastasis in the brain $(p<0.0001$ and $p=0.0004$, respectively), normal LDH levels ( $p<0.0001$ and $p=0.0063$, respectively), ECOG -0 (for both $p<0.0001$ ), lower stage ( $p<0.0001$ and $p=0.0027$, respectively), less than three locations of metastatic lesions ( $p=0.0001$ and $p=0.0031$, respectively), objective response (complete response + partial response) to the treatment (for both $p<0.0001)$, more than one treatment line $(p=0.0072$ and $p=0.0432$, respectively), and termination of the treatment due to toxicity ( $p<0.0001$ and $p=0.0001$, respectively). Chemotherapy as a treatment regimen in the first line had the worst effect on PFS ( $p=0.0035)$, but without affecting OS $(p=0.1336)$.

Other variables, including gender, age $>65$ years, location of the primary lesion, and the presence of BRAF mutation had no statistically significant effect on OS and PFS.

Multivariate analyses confirmed that normal LDH levels, the absence of brain metastasis, ECOG 0, and objective response to treatment were strong predictors of longer OS. For PFS, the absence of brain metastasis, very good general condition, and response to treatment were found to be predictive factors. Table 3 presents the results of the multivariate analyses.

\section{Results and discussion}

In the present study, we have indicated current trends and treatment effects of patients with advanced/disseminated melanoma through real-world results. The presented results confirm the effectiveness of these therapies.

The survival outcomes in a similar population of patients treated between 1995 and 2005 at the National Research Institute of Oncology in Warsaw and Krakow, before the discovery and approval of new therapies, were worse [5]. The median OS and PFS were 7.1 and 3.5 months, respectively, with one-year and two-year survival rates of $32 \%$ and $12.5 \%$, respectively [5]. In our study, patients had dramatically better treatment results: median OS and PFS were 14.9 and 6.7 months, respectively, and the one-year and two-year survival rates were 52\% and $22 \%$, respectively. It has been found that the number of responses to treatment is higher when new therapies are used. In our study, 43\% ORR and 77\% DCR were noted, and 19\% ORR and 65\% DCR were recorded in the 1995-2005 study. All the results obtained in our study are comparable with the treatment outcomes via new therapies in other centres in Poland [15-18] and other studies with real-world data [19-22].

The most unexpected finding in our study is the correlation between the type of first-line treatment and OS. Across the experimental group, patients received IT, TT, and $\mathrm{CTH}$ as a first-line treatment, and the median OS was 19.2, 12.6, and 15.9 months, respectively. The medi- 
an OS was longer in the CTH group than in the TT group and shorter than in the IT group. This could be because the group of patients receiving $\mathrm{CTH}$ had better prognostic factors than patients receiving IT or $\mathrm{TT}$. In the $\mathrm{CTH}$ group, only $9 \%$ of patients had brain metastases and $26 \%$ exhibited elevated LDH than in the group receiving IT (15\% and $39 \%$, respectively) or TT (36\% and $62 \%$, respectively). However, the most interesting finding is that each patient who received $\mathrm{CTH}$ in the first-line treatment qualified for the second-line treatment - either IT ( $81 \%$ of patients) or TT (19\% of patients). Therefore, the CTH-IT or CTH-TT treatment group is a very special group. It should also be noted that patients in a good or very good condition more often qualified for the second-line treatment (this is due to the inclusion criteria in the drug program with IT or TT). Conclusions from real-world data in the United Kingdom (UK) for ipilimumab were also similar [19]. The UK study also revealed that patients in a better condition and with favourable prognostic factors had a better prognosis [19]. Hence, new therapies (especially IT) are active and effective in subsequent treatment lines.

Another important finding is that patients with more than one treatment line had a better prognosis. It should be noted that the median PFS in the second and third lines were similar, at 3.1 and 3.9 months, respectively. Also, in a real-world study, Cimminiello et al. revealed the efficacy of subsequent treatments [20]. Hence, the third or fourth lines of treatment in patients with advanced melanoma could be beneficial and should be considered as a treatment option. However, this hypothesis should be tested in prospective clinical trials.

Another observation is the difference in OS, PFS, and treatment responses between IT and TT in the first and second lines of treatment. A comparison of IT and TT treatment outcomes in the first and second lines indicated that patients receiving IT in the second line had a worse prognosis than patients receiving IT treatment in the first line. Existing studies indicate that immunotherapy administered before the targeted therapy may provide better results even in patients with BRAF mutation [23-27]. In our analysis, better prognosis in the first-line treatment with IT could be due to a lower number of patients receiving TT in the second line. However, $50 \%$ of our patients continued treatment with IT in the second line (received ipilimumab, anti-CTLA-4), which also affected the obtained results.
However, this sequence of the treatment requires further research and observation.

Another observation is the difference in OS between $B R A F$-mutated and BRAF-wild type populations in first-line IT treatment. This may be the result of selecting BRAF-mutated patients for IT because BRAF-mutated patients who had better prognostic factors were more often qualified for IT treatment in the first line.

Our analysis has certain limitations because it is a retrospective study. Nevertheless, the experimental group is representative and comparable with other groups with respect to prognostic factors. The presented analysis (2013-2019) has indicated that normal LDH levels, absence of brain metastases, and good performance status have a positive impact on prognosis. Other studies with real-world data have also revealed similar prognostic factors [19-22]. Similar results were also found in research conducted before the era of new therapies [28-33] and in the analysis from 1995-2005 [5], which prove the similarity between these two populations. As expected, our data indicated that the presence of brain metastases predicts worse OS and PFS, which supports recently published studies $[34,35]$. However, older analyses did not reveal such a strong impact of brain involvement on OS and PFS [3-5, 36-38], which is probably due to the absence of neuroimaging techniques in the detection of brain metastases. In our analysis, the prognostic factors associated with OS are similar to those obtained in other analyses and Melanoma Staging: American Joint Committee on Cancer (AJCC) $8^{\text {th }}$ Edition [35, 39]. It should also be noted that despite the use of new treatment options and improved survival rates, prognostic factors remain the same, and the same group of patients still has a poor prognosis (poor general condition, brain metastases, and elevated LDH).

\section{Conclusions}

The administration of new therapies for the treatment of patients with advanced/disseminated melanoma significantly prolonged survival in this group of patients. Nevertheless, further studies should be conducted to assess the effectiveness of various sequences of treatment.

The authors declare no conflict of interest.

Table 3. Results of the multivariate Cox proportional hazards models in metastatic melanoma patients before first-line therapy

\begin{tabular}{|c|c|c|c|c|c|c|c|}
\hline \multirow[t]{2}{*}{ Parameter } & \multirow[t]{2}{*}{ Category } & \multicolumn{3}{|c|}{ OS } & \multicolumn{3}{|c|}{ PFS } \\
\hline & & $p$-value & HR & $95 \% \mathrm{Cl}$ & $p$-value & HR & $95 \% \mathrm{Cl}$ \\
\hline Brain metastasis, no vs. yes & No & $<0.0001$ & 0.39 & $0.3-0.5$ & 0.0019 & 0.60 & $0.4-0.8$ \\
\hline LDH, normal vs. elevated & Normal & 0.0005 & 0.57 & $0.4-0.8$ & 0.1811 & 0.83 & $0.6-1.1$ \\
\hline ECOG, 0 vs. 1 vs. $>1$ & ECOG 1 & 0.4328 & 0.48 & $0.3-0.8$ & 0.4693 & 0.63 & $0.4-1.0$ \\
\hline ECOG, 0 vs. 1 vs. $>1$ & ECOG 0 & $<0.0001$ & 0.16 & $0.1-0.4$ & 0.0012 & 0.30 & $0.1-0.6$ \\
\hline$C R+P R$, vs. SD vs. PD & $C R+P R$ & $<0.0001$ & 0.26 & $0.2-0.4$ & $<0.0001$ & 0.08 & $0.1-0.1$ \\
\hline$C R+P R$, vs. SD vs. PD & SD & 0.3933 & 0.45 & $0.3-0.7$ & 0.0071 & 0.19 & $0.1-0.3$ \\
\hline
\end{tabular}

PFS - progression-free survival, OS - overall survival, IT - immunotherapy, TT - targeted therapy, CHT - chemotherapy, LDH - lactate dehydrogenase, ECOG/PS Eastern Cooperative Oncology Group/Performance status, $C R$ - complete response, PR - partial response, SD - stable disease, $P D$ - progression of disease 


\section{References}

1. Barth A, Wanek LA, Morton DL Prognostic factors in 1521 melanoma patients with distant metastases. J Am Coll Surg 1995; 181: 193-201.

2. Del Prete SA, Maurer LH, O’Donnell J, Forcier RJ, LeMarbre P. Combination chemotherapy with cisplatin, carmustine, dacarbazine, and tamoxifen in metastatic melanoma. Cancer Treat Rep 1984; 68: 14031405.

3. Lattanzi SC, Tosteson T, Chertoff J, et al. Dacarbazine, cisplatin and carmustine, with or without tamoxifen, for metastatic melanoma: 5-year follow-up. Melanoma Res 1995; 5: 365-369.

4. Chapman PB, Einhorn LH, Meyers ML, et al. Phase III multicenter randomized trial of the Dartmouth regimen versus dacarbazine in patients with metastatic melanoma. J Clin Oncol 1999; 17: 2745-2751.

5. Cybulska-Stopa B, Skoczek M, Ziobro M, et al. Results of systemic treatment of cutaneous melanoma in inoperable stage III and IV. Contemp Oncol (Pozn) 2012; 16: 532-545.

6. Robert C, Long GV, Brady B, et al. Nivolumab in previously untreated melanoma without BRAF mutation. N Engl J Med 2015; 372: 320-330.

7. Ascierto PA, Long GV, Robert C, et al. Survival Outcomes in Patients With Previously Untreated BRAF Wild-Type Advanced Melanoma Treated With Nivolumab Therapy: Three-Year Follow-up of a Randomized Phase 3 Trial. JAMA Oncol 2019; 5: 187-194.

8. Long GV, Schachter J, Ribas A, et al. 4-year survival and outcomes after cessation of pembrolizumab (pembro) after 2-years in patients (pts) with ipilimumab (ipi)-naïve advanced melanoma in KEYNOTE-006. J Clin Oncol 2018; 36: 9503-9503.

9. Robert C, Ribas A, Schachter J, et al. Pembrolizumab versus ipilimumab in advanced melanoma (KEYNOTE-006): post-hoc 5-year results from an open-label, multicentre, randomised, controlled, phase 3 study. Lancet Oncol 2019; 20: 1239-1251.

10. Wolchok JD, Kluger H, Callahan MK, et al. Nivolumab plus ipilimumab in advanced melanoma. N Engl J Med 2013; 369: 122-133.

11. Dummer R, Ascierto PA, Gogas HJ, et al. Encorafenib plus binimetinib versus vemurafenib or encorafenib in patients with BRAF-mutant melanoma (COLUMBUS): a multicentre, open-label, randomised phase 3 trial. Lancet Oncol 2018; 19: 603-615.

12. Dummer R, Ascierto PA, Gogas HJ, et al. Overall survival in patients with BRAF-mutant melanoma receiving encorafenib plus binimetinib versus vemurafenib or encorafenib (COLUMBUS): a multicentre, open-label, randomised, phase 3 trial. Lancet Oncol 2018; 19: 1315-1327.

13. Mackiewicz J, Mackiewicz A. Programmed cell death 1 checkpoint inhibitors in the treatment of patients with advanced melanoma. Contemp Oncol (Pozn) 2017; 21: 1-5.

14. Cybulska-Stopa B, Świtaj T, Koseła-Paterczyk H. Combined or sequential treatment of advanced melanoma? Nowotwory 2019; 69: 125-132.

15. Brzozowska M, Wierzba W, Śliwczyński A, et al. Analysis of the survival of patients receiving systemic treatment for melanoma of the skin: a retrospective population study with patients treated in Poland in 2011-2015. Postepy Dermatol Alergol. 2019; 36: 438-441.

16. Rutkowski P, Kozak K, Mackiewicz J, et al. The outcomes of Polish patients with advanced BRAF-positive melanoma treated with vemu rafenib in a safety clinical trial. Contemp Oncol (Pozn) 2015; 19: 280-283.

17. Wiater K, Świtaj T, Mackiewicz J, et al. Efficacy and safety of ipilimumab therapy in patients with metastatic melanoma: a retrospective multicenter analysis. Contemp Oncol (Pozn) 2013; 17: 257-262.

18. Brzozowska M, Wierzba W, Śliwczyński A, Świerkowski M, Potemski P, Marczak M. Analysis of survival of patients treated with vemurafenib, ipilimumab and dabrafenib for advanced skin melanoma in daily clinical practice (Real-World Data): retrospective analysis of patients treated under drug/reimbursement programmes in Poland in 20132016. Melanoma Res 2018; 28: 52-55.

19. Ahmad SS, Qian W, Ellis S, et al. Ipilimumab in the real world: the UK expanded access programme experience in previously treated advanced melanoma patients. Melanoma Res 2015; 25: 432-442.

20. Cimminiello C, Indini A, Di Guardo L, Prisciandaro M, Randon G, Tolomio E, De Braud F, Del Vecchio M. Pembrolizumab in the treatment of ad vanced/metastatic melanoma a single-center institution experience. Melanoma Res 2019; 29: 289-294.

21. Schouwenburg MG, Jochems A, Leeneman B, et al. Vemurafenib in BRAF-mutant metastatic melanoma patients in real-world clinical practice prognostic factors associated with clinical outcomes. Melanoma Res 2018; 28: 326-332.
22. Mangana J, Cheng PF, Kaufmann C, et al. Multicenter, real-life experience with checkpoint inhibitors and targeted therapy agents in advanced melanoma patients in Switzerland. Melanoma Res 2017; 27: 358-368.

23. Wu M, Wang Y, Xu Y, et al. Indirect comparison between immune checkpoint inhibitors and targeted therapies for the treatment of melanoma. J Cancer 2019; 10: 6114-6123.

24. Schilling B, Martens A, Geukes Foppen MH, et al. First-line therapy-stratified survival in BRAF-mutant melanoma: a retrospective multicenter analysis. Cancer Immunol Immunother 2019; 68: 765-772.

25. Ascierto PA, Simeone E, Giannarelli D, Grimaldi AM, Romano A, Mozzillo $N$. Sequencing of BRAF inhibitors and ipilimumab in patients with metastatic melanoma: a possible algorithm for clinical use. J Transl Med 2012; 10: 107.

26. Ackerman A, Klein O, McDermott DF, et al. Outcomes of patients with metastatic melanoma treated with immunotherapy prior to or after BRAF inhibitors. Cancer 2014; 120: 1695-1701.

27. Johnson DB, Pectasides E, Feld E, et al. Sequencing Treatment in BRAFV600 Mutant Melanoma: Anti-PD-1 Before and After BRAF Inhibition. J Immunother 2017; 40: 31-35.

28. Presant CA, Bartolucci AA. Prognostic factors in metastatic malignant melanoma: The Southeastern Cancer Study Group Experience. Cancer 1982; 49: 2192-2196.

29. Balch CM, Soong SJ, Murad TM, Smith JW , Maddox WA, Durant JR. A multifactorial analysis of melanoma IV. Prognostic factors in 200 melanoma patients with distant metastases (stage III). J Clin Oncol 1983; 1: $126-134$.

30. Eton O, Legha SS, Moon TE, et al. Prognostic factors for survival of patients treated systemically for disseminated melanoma. J Clin Oncol 1998; 16: 1103-1111.

31. Sirott MN, Bajorin DF, Wong GY, Tao Y, Chapman PB, Templeton MA Houghton AN. Prognostic factors in patients with metastatic malignant melanoma. A multivariate analysis. Cancer 1993; 72: 3091-3098.

32. Unger JM, Flaherty LE, Liu PY, Albain KS, Sondak VK. Gender and other survival predictors in patients with metastatic melanoma on Southwest Oncology Group trials. Cancer 2001; 91: 1148-1155.

33. Bhatia S, Tykodi S, Thompson J. Treatment of metastatic melanoma: Overview. Oncology 2009; 23960: 488-496.

34. Drago JZ, Lawrence D, Livingstone E, et al. Clinical experience with combination BRAF/MEK inhibitors for melanoma with brain metastases: a real-life multicenter study. Melanoma Res 2019; 29: 65-69.

35. Gershenwald JE, Scolyer RA. Melanoma Staging: American Joint Committee on Cancer (AJCC) 8th Edition and Beyond. Ann Surg Oncol 2018; 25: 2105-2110.

36. Bhatia S, Tykodi S, Thompson J. Treatment of metastatic melanoma: Overview. Oncology 2009; 23960: 488-496.

37. Atkins MB, Kunkel L, Sznol M, Rosenberg SA. High-dose recombinant interleukin-2 therapy in patients with metastatic melanoma: long-term survival update. Cancer J Sci Am 2000; 6 (Suppl 1): S11-14.

38. Atkins MB, Lotze MT, Dutcher JP, et al. High-dose recombinant interleukin 2 therapy for patients with metastatic melanoma: analysis of 270 patients treated between 1985 and 1993. J Clin Oncol 1999; 17: 21052116.

39. Teterycz P, Ługowska I, Koseła-Paterczyk H, Rutkowski P. Comparison of seventh and eighth edition of AJCC staging system in melanomas at locoregional stage. World J Surg Oncol 2019; 17: 129.

\section{Address for correspondence}

\section{Bożena Cybulska-Stopa}

Clinical Oncology Department

Maria Sklodowska-Curie National Research Institute of Oncology

Krakow Branch

11 Garnacarska St.

31-115 Krakow, Poland

e-mail: bcybulskastopa@vp.pl

Submitted: 4.04 .2020

Accepted: 11.05 .2020 